\title{
Roles of Serine Protease Inhibitor Kazal type 1 (SPINK1) in Prostate Cancer
}

Chengwu Shen ${ }^{1}$, Jing Zhang ${ }^{1}$, Mei Qi ${ }^{2}$, Yannicca WYChang ${ }^{3}$ and Bo Han ${ }^{2,4 *}$

${ }^{1}$ Department of Pharmacy, Shandong Provincial Hospital, Jinan 250021 China

${ }^{2}$ Department of Pathology, School of Medicine, Shandong University, Jinan 250012, China 3Department of Health and Disease and Psychology, University of Tornoto,

Markham, Canada 4Department of Pathology, Qilu Hospital, Shandong University, Jinan 250012, China

\begin{abstract}
Altered genes that play a driving role in cancer development can often serve as specific diagnostic markers, criteria of molecular classification and therefore potential therapeutic targets. Serine protease inhibitor Kazal type 1 (SPINK1), also known as pancreatic secretory trypsin inhibitor or tumor-associated trypsin inhibitor, encodes a 56 amino acid secreted peptide, and its normal function is thought to be the inhibition of serine proteases such as trypsin. Recent studies have indicated marked overexpression of SPINK1 defines an aggressive molecular subtype of ETS (erythroblastosis virus E26 transformation-specific) fusion-negative prostate cancer ((PCa) patients. SPINK1 may act as an autocrine growth factor and promotes PCa growth and invasion. Most recently, we suggested that SPINK1 induces epithelial-mesenchymal transition (EMT) through EGFR signaling pathway in PCa. The association between SPINK1 overexpression and poor prognosis in PCa has been reported. Notably, SPINK1 might be a novel extracellular therapeutic target in a subset of high-grade PCa patients. In this review, we will summarize the current understanding of SPINK1 involving its role in PCa biology, association with prognosis as well as perspective in therapy from the pathologist's point of view.
\end{abstract}

Keywords: Serine protease inhibitor kazal type 1; Prostate cancer; ETS; Prognosis; Target

Prostate cancer $(\mathrm{PCa})$ is the most commonly diagnosed non-skin cancer in American men. It is estimated that 233,000 Americans will be diagnosed with PCa in 2014 and over 29,000 men will die from the disease [1]. PCa is a common heterogeneous disease with only a small fraction of patients will progress rapidly and require immediate treatment [2]. Consequently, two major clinical challenges were posed by the current inability to readily distinguish indolent from aggressive tumors in PCa patients and development of novel therapeutic strategies targeting aggressive tumors.

Altered genes that play a driving role in cancer development can often serve as specific diagnostic markers, criteria of molecular classification and therefore potential therapeutic targets [3]. The recent finding of overexpression of serine protease inhibitor Kazal type 1 (SPINK1) may represent a novel therapeutic target in selected PCa patients. In this review, we will summarize the structure of SPINK1 protein, current understanding of its role in PCa biology, association with prognosis as well as perspective in therapy from the pathologist's point of view.

\section{An overview of SPINK1}

SPINK1, also known as pancreatic secretory trypsin inhibitor or tumor-associated trypsin inhibitor, encodes a 56 amino acid secreted peptide, which contains three disulfide bonds and a trypsin-specific binding site formed by Lys-Ile [4,5]. SPINK1 is usually with a signal peptide of approximately 20 amino acids at N-terminal. Analysis of the human SPINK1 gene reveals that it is located on $5 \mathrm{q} 32$ containing approximately $7.5 \mathrm{~kb}$ and four exons. A $40 \mathrm{bp}$ DNA fragment located between $\mathrm{kb}-3.84$ and -3.80 carries the element responsible for both transcriptional activity and IL-6-induced gene expression [4]. SPINK1 was originally isolated from the pancreas and its primary function is inhibition of serine proteases, such as trypsin, in the pancreas and small intestines [6]. Multiple variants of SPINK1 have been previously shown to be a strong causal factor for chronic pancreatitis possibly due to inactivation of trypsin $[7,8]$. Additionally, inactivation of SPINK1 in the pancreas results in autophagy of exocrine pancreatic cells, suggesting that SPINK1 may be important in cell survival. In liver cells, expression of SPINK1 can be induced by Hepatitis B virus and protects liver cells from serine protenase dependent cellular apoptosis [4]. Collectively,
SPINK1 appears to play an important role in both cell survival and prevention of apoptosis by several different pathways in normal tissues.

Overexpression of SPINK1 has been observed in multiple human malignancies, including cancers of the colon, breast, liver and urinary bladder [9-12]. Notably, increased SPINK1 expression was associated with liver metastasis and was an independent predictor of poor prognosis in cancer of the colon and the breast [10,12]. Lee et al. concluded that SPINK1 overexpression contributed to cell growth advantage and enhanced the metastatic potential of tumors and suggested using SPINK1, AFP and osteopontin as combined markers for the prediction of early tumor recurrence of hepatocellular carcinoma [13].

\section{Expression and the Biological Role of SPINK1 in Prostate Cancer}

In 2007, Paju et al. first reported SPINK1 can be overexpressed in PCa tissues and cell line 22RV1 [14]. (In 2008, using a Cancer Outlier Profile Analysis (COPA) strategy, Tomlins et al identified SPINK1 outlier expression exclusively in ETS (Erythroblastosis virus E26 transformation-specific) rearrangement negative PCa patients $(\sim 10 \%$ of total cases) [15]. Gene fusions involving ETS family transcription factors are present in approximately $50 \%$ of prostate-specific antigen (PSA)-screened localized PCas and in $15-30 \%$ of population-based cohorts [16]. Recent work has demonstrated that prostate tumors can be stratified by dominant genetic alterations, such as chromosomal rearrangements involving ETS family transcription factors [17]. In surgically resected Pca patients, Lippolis et al. [18] and Bismar et al. [19] confirmed exclusivity of SPINK1 overexpression and ETS gene fusions, but that is not the case in endocrine-treated patients [20]. In line with

*Corresponding author: Bo Han, Department of Pathology, Shandong University Medical School, \#44, Wenhua Xi Rd, Jinan, 250012 P.R. China, Tel: +86-53188382574; E-mail: boh@sdu.edu.cn

Received July 23 2014; Accepted October 06, 2014; Published October 08, 2014

Citation: Shen C, Zhang J, Qi M, Chang YWY, Han B (2014) Roles of Serine Protease Inhibitor Kazal type 1 (SPINK1) in Prostate Cancer. Med chem 4: 725728. doi:10.4172/2161-0444.1000220

Copyright: ( $) 2014$ Shen C, et al. This is an open-access article distributed under the terms of the Creative Commons Attribution License, which permits unrestricted use, distribution, and reproduction in any medium, provided the original author and source are credited. 
these studies, we also suggested SPINK1 is mutually exclusive with ERG rearrangement status in Chinese PCa patients and the prevalence of SPINK1 overexpression is comparable with that of western populations [21]. In contrast, some previous reports did not find such mutual exclusivity, although the majority of which suggested the incidence rate was very low for concomitance of SPINK1 and ETS fusions [20,22]. In a most recent follow-up study including $879 \mathrm{PCa}$ patients treated by radical prostatectomy, Flavin et al. [23] reported that SPINK1 protein expression in $8 \%(74 / 879)$ of cases. Additionally, they showed SPINK1 over-expression was seen in 47 of 427 (11\%) ERG negative samples and in 19 of 427 (4\%) ERG positive cases. In an attempt of characterizing SPINK1 expression in uncommon histolgic variant, we showed SPINK1 was expressed in 6\% of ETS negative histologic variants and specifically in ductal adenocarcinoma [24]. The discrepancy might be partially explained by cohort design, patients' race, antibody selection and criteria for SPINK1 IHC positivity.

The molecular mechanism underlying SPINK1 overexpression remains unclear. Using FISH analysis, Tomlins et al. demonstrated no evidence of gene amplification or genetic arrangement, respectively, in samples with SPINK1 overexpression [15]. Thus, SPINK1 may be activated by increased transcription activity. Alternatively, SPINK1 may be activated by a unique upstream genetic event [15]. So far, research data regarding this field is still lacking.

Currently, in vitro and in vivo data suggested that SPINK1 is oncogenic in PCa $[15,25]$. Tomlins et al. demonstrated that SPINK1 knockdown in 22RV1 cells attenuates invasion [15]. In addition, Ateeq et al. suggested that recombinant SPINK1 protein (rSPINK1) stimulates cell proliferation in benign RWPE as well as cancerous prostate cells [25]. Of note, RWPE cells treated with either rSPINK1 or conditioned medium from 22RV1 PCa cells (SPINK1+/ETS-) significantly increased cell invasion when compared with untreated cells. In contrast, knockdown of SPINK1 in 22RV1 cells inhibited cell proliferation, cell invasion, and tumor growth in xenograft assays [25].

It remains unknown how SPINK1 contributes to PCa carcinogenesis and tumor progression. The expression of SPINK1 is invariably associated with expression of tumor-associated trypsin, which activates several matrix metalloproteinases. Several reports have suggested that the association between SPINK1 expression and adverse prognosis in cancer has been ascribed to the expression of trypsin by the tumors $[6,26]$. However, oncogenic effect of SPINK1 seemed to be independent of trypsin activity in PCa [25]. Indeed, recent studies have focused attention on the interaction of SPINK1 and epidermal growth factor receptor (EGFR) [11,25,27]. SPINK1 and EGF, the EGFR ligand, share $50 \%$ amino acid homology and several structural similarities [28]. Similar to EGF, Ozaki et al. [29] have showed that SPINK1 is able to phosphorylate EGFR and its downstream molecules including STAT3, Akt and ERK in pancreatic cancer cells. Ateeq et al. [25] proposed that SPINK1 functions at least in part to stimulate EGFR signaling in an autocrine loop. Clinically, the notion of SPINK1-EGFR axis was evidenced by the significant co-overexpression of SPINK1 and EGFR in PCa patients cohort [21]. Most recently, we reported that SPINK1 induces epitheial mesenchymal transition (EMT) in PCa via activating EGFR, leading to increased migratory and invasive capacity in vitro [21]. EGFR/MAPK pathway was mainly involved in this process. Connective tissue growth factor might be a main downstream molecule of this pathway. Of note, EMT is an early embryonic development program and plays a critical role in cancer progression and metastasis [30]. Thus, our data may partially explain the mechanism by which SPINK1 promotes PCa progression.

\section{Prognostic Association of SPINK1 Overexpression}

Initial work in ETS rearrangement negative PCas indicated overexpression of SPINK1 defines a distinct molecular subtype with an aggressive phenotype [15]. Compared with other patients, SPINK1positive PCa patients may have a worse Gleason score and a higher risk of disease recurrence, and poor prognosis. Later, Leinonen et al. [20] reported that SPINK1 expression is associated with aggressive form of the disease and could serve as a biomarker in endocrine-treated PCa patients. In consistent with these findings, our results revealed that SPINK1 overexpression is an unfavorable prognostic factor in Chinese PCa patients [21].

Conversely, there are several studies that have reported an absence of such a clinical correlation between the SPINK1 overexpression and outcome. In the study performed by Lippolis et al. [18], SPINK1 was weakly associated with pathological tumor stage, but not associated with biochemical recurrence or development of metastatic disease. Grupp et al. [31] found no association between SPINK1 status and clinico-pathologic factors or biochemical recurrence in a large cohort of surgically treated PCa patients. Similarly, Flavin et al. [23] found no positive association between SPINK1 status and prostate cancerspecific survival. Taken together, prognostic significance of SPINK1 overexpression in PCa merits further investigation.

\section{SPINK1: A Potential Therapeutic Target for Prostate Cancer}

The goal of target therapy is to treat patients individually by targeting the pathways that are specifically present or activated in that specific tumor. Monoclonal antibody treatments have been effective against proteins that are presented outside of the cell, such as trastuzumab in breast cancers and rituximab for B cell lymphomas that express CD20 [32]. Accordingly, small-molecule inhibitors have been used to interfere with signaling networks inside of cells (Figure 1). For instance, Gleevec is a small-molecule inhibitor that can be used to treat a subset of chronic myelogenous leukemia, which is driven by BCR-ABL [33]. So far, several lines of evidence have suggested that SPINK1 may be a potential novel target for PCa treatment. First, SPINK1 is an excellent "druggable" target. Although recurrent gene fusions involving ETS family transcription factors have been identified in $\sim 50 \%$ of the PCa patients in western countries, their products are considered poor therapeutic targets by conventional pharmaceutical approaches, owing to lack of enzymatic activity, inaccessibility in the nucleus and requirement of interaction with other proteins to achieve specificity. SPINK1 protein is a protease inhibitor, which can be

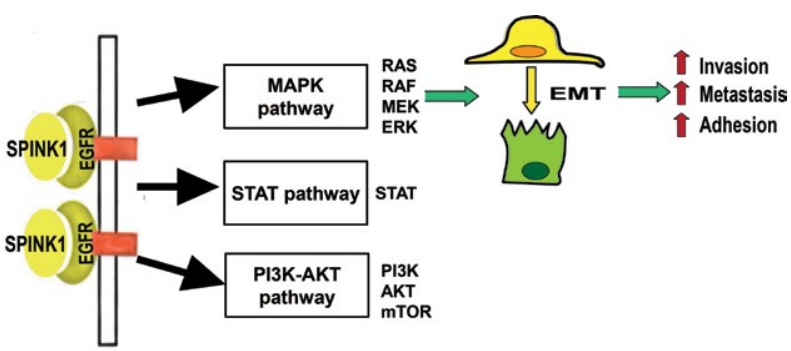

Figure 1: SPINK1 promotes epithelial-mesenchymal transition (EMT) through EGFR signaling pathway in PCa as a growth factor.

SPINK1 binds to EGFR as a autocrine growth factor. There are at least three signaling pathways downstream of EGFR, including the PI3K/AKT, JAK/STAT, and MAPK/MEK/ERK signaling pathway. All three downstream targets are phosphorylated by SPINK1 and EGF. However, the MAPK/MEK/ ERK pathway is actually mainly involved in SPINK1-induced epithelialmesenchymal transition (EMT). 
secreted into the extracellular matrix [34]. Of note, SPINK1 may be secreted by the same cell that it stimulates, and thus it can be classified as an autocrine growth factor [25]. In vivo experiments have confirmed it to be druggable [25], and because of the effect of antibodies against SPINK1 on prostate, colon, and breast cancer xenografts, it has been suggested that humanized monoclonal antibodies to SPINK1 should be developed for cancer therapy [11]. Secondly, in vivo data showed that administration of a monoclonal antibody to SPINK1 reduced cellular proliferation and the invasion and growth of SPINK1-producing 22RV1 tumor xenograft [25]. Interestingly, this inhibitory effect was enhanced by co-administration of EGFR antibody (Cetuximab) [25].

In recent years, EGFR was proposed as a promising candidate target since about $18 \%$ to $40 \%$ of advanced PCa patients showed high EGFR expression [35]. However, in a multi-center clinical trial, the use of cetuximab and other EGFR antagonists in the treatment of advanced PCa patients did not achieve the desired effect [36]. Interestingly, a response has been observed in only about $10 \%$ of the advanced PCa cases with treatment of Cetuximab and other EGFR antagonists. Of note, this is the proportion of PCas that show increased SPINK1 production; however, it is not known whether the response is associated with SPINK1 expression and whether combined use of EGFR inhibitors and SPINK1 antibodies would improve the response.

It is likely that humanized SPINK1 antibodies to be used for treatment of PCa are under development [11]. Patients suitable for such treatment need to be selected on the basis of SPINK1 expression.

\section{Challenges and Perspectives}

Although humanized monoclonal antibodies are emerging as a new therapeutic strategy for various malignancies, the development of therapeutic antibodies requires a deep understanding of cancer serology, protein-engineering techniques, mechanisms of action and resistance, the interplay between the immune system and cancer cells, and presence of potential adverse side effects [37]. In PCa, SPINK1 has potential applications in the prognosis and therapeutic target selection on cancer patients. However, there are several issues to be solved in the forefront. Firstly, why SPINK1 is overexpressed in a subset of ETS fusion negative PCa cases? It is hypothesized that an unknown molecular lesion that initially drives ETS fusion negative tumors may predispose to activation of SPINK1 overexpression [25]. Secondly, what is the exact link between SPINK1 overexprssion and PCa invasion and progression? Do any other novel molecular mechanisms exist besides SPINK1-EGFR axis? Thirdly, and most importantly, is SPINK1-targeted therapy effective to the subset of high grade PCa cases with SPINK1 overexpression? How to design the molecules against SPINK1 protein and what is the best delivery strategy? We believe the answers to above questions would pave the way for better diagnostic and/or therapeutic modalities of SPINK1+ advanced PCa patients.

\section{Acknowledgments}

Supported by the National Natural Science Foundation of China (Grant No. 81171951), the Independent Innovation Foundation to Universities and Colleges of Shandong Province, Scientific Research Foundation for Returned Scholars, Ministry of Education of China.

\section{References}

1. Siegel R, Ma J, Zou Z, Jemal A (2014) Cancer statistics, 2014. CA Cancer J Clin 64: 9-29.

2. Shen MM, Abate-Shen C (2010) Molecular genetics of prostate cancer: new prospects for old challenges. Genes Dev 24: 1967-2000.

3. Vogelstein B, Kinzler KW (2004) Cancer genes and the pathways they control. Nat Med 10: 789-799.
4. Wang GP, Xu CS (2010) Pancreatic secretory trypsin inhibitor: More than a trypsin inhibitor. World J Gastrointest Pathophysiol 1: 85-90.

5. Ohmuraya M, Yamamura K (2011) Roles of serine protease inhibitor Kazal type 1 (SPINK1) in pancreatic diseases. ExpAnim 60: 433-444.

6. Paju A, Stenman UH (2006) Biochemistry and clinical role of trypsinogens and pancreatic secretory trypsin inhibitor. Crit Rev Clin Lab Sci 43: 103-142.

7. Bagul A, Pushpakom S, Balachander S, Newman WG, Siriwardena A (2009) The SPINK1 N34S variant is associated with acute pancreatitis. Eur J Gastroenterol Hepatol 21: 485.

8. Derikx MH, Szmola R, teMorsche RH, Sunderasan S, Chacko A, et al. (2009) Tropical calcific pancreatitis and its association with CTRC and SPINK1 (p.N34S) variants. Eur J Gastroenterol Hepatol 21: 889-894.

9. Gaber A, Johansson M, Stenman UH, Hotakainen K, Ponten F, et al. (2009) High expression of tumour-associated trypsin inhibitor correlates with liver metastasis and poor prognosis in colorectal cancer. $\mathrm{Br} \mathrm{J}$ Cancer 100: 15401548.

10. Gaber A,Nodin B, Hotakainen K, Nilsson E,Stenman UH,et al. (2010) Increased serum levels of tumour-associated trypsin inhibitor independently predict a poor prognosis in colorectal cancer patients. BMC Cancer 10: 498.

11. Stenman UH (2011) SPINK1: a new therapeutic target in cancer? Clin Chem 57: $1474-1475$.

12. Soon WW, Miller LD, Black MA,Dalmasso C, Chan XB, et al.(2011) Combined genomic and phenotype screening reveals secretory factor SPINK1 as an invasion and survival factor associated with patient prognosis in breast cancer. EMBO Mol Med 3: 451-464.

13. Lee YC, Pan HW, Peng SY, Lai PL, Kuo WS,et al. (2007) Overexpression of tumour-associated trypsin inhibitor (TATI) enhances tumour growth and is associated with portal vein invasion, early recurrence and a stage-independent prognostic factor of hepatocellular carcinoma. Eur J Cancer 43: 736-744.

14. Paju A, Hotakainen K, Cao Y, Laurila T, Gadaleanu V, et al. (2007) Increased expression of tumor-associated trypsin inhibitor, TATI, in prostate cancer and in androgen-independent 22Rv1 cells. EurUrol 52: 1670-1679.

15. Tomlins SA, Rhodes DR, Yu J,Varambally S,Mehra R,et al. (2008) The role of SPINK1 in ETS rearrangement-negative prostate cancers. Cancer Cell 13: 519-528.

16. Kumar-Sinha C,Tomlins SA, Chinnaiyan AM (2008) Recurrent gene fusions in prostate cancer. Nat Rev Cancer 8: 497-511.

17. Rubin MA, Maher CA, Chinnaiyan AM (2011) Common gene rearrangements in prostate cancer. J Clin Oncol 29: 3659-3668

18. Lippolis G, Edsjo A, Stenman UH, Bjartell A (2013) A high-density tissue microarray from patients with clinically localized prostate cancer reveals ERG and TATI exclusivity in tumor cells. Prostate Cancer Prostatic Dis 16: 145-150.

19. Bismar TA, Yoshimoto M, Duan Q, Liu S, Sircar K, et al. (2012) Interactions and relationships of PTEN, ERG, SPINK1 and AR in castration-resistant prostate cancer. Histopathology 60: 645-652.

20. Leinonen KA, Tolonen TT, Bracken H, Stenman UH, Tammela TL, et al. (2010) Association of SPINK1 expression and TMPRSS2:ERG fusion with prognosis in endocrine-treated prostate cancer. Clin Cancer Res 16: 2845-2851.

21. Wang C, Wang L, Su B, Lu N, Song J, et al. (2014) Serine protease inhibitor Kazal type 1 promotes epithelial-mesenchymal transition through EGFR signaling pathway in prostate cancer. Prostate 74: 689-701.

22. Bhalla R, Kunju LP, Tomlins SA, Christopherson K, Cortez C, et al. (2013) Nove dual-color immunohistochemical methods for detecting ERG-PTEN and ERGSPINK1 status in prostate carcinoma. Mod Pathol 26: 835-848.

23. Flavin R, Pettersson A, Hendrickson WK, Fiorentino M, Finn S, et al. (2014) SPINK1 Protein Expression and Prostate Cancer Progression. Clin Cancer Res 20: 4904-4911.

24. Han B, Mehra R, Suleman K, Tomlins SA, Wang L, et al. (2009) Characterization of ETS gene aberrations in select histologic variants of prostate carcinoma. Mod Pathol 22: 1176-1185.

25. Ateeq B, Tomlins SA, Laxman B, Asangani IA, Cao Q, et al. (2011) Therapeutic targeting of SPINK1-positive prostate cancer. Sci Transl Med 3: 72ra17.

26. Paju A, Sorsa T, Tervahartiala T, Koivunen E, Haglund C, et al. (2001) The levels of trypsinogenisoenzymes in ovarian tumour cyst fluids are associated with promatrix metalloproteinase-9 but not promatrix metalloproteinase-2 activation. Br J Cancer 84: 1363-1371. 
Citation: Shen C, Zhang J, Qi M, Chang YWY, Han B (2014) Roles of Serine Protease Inhibitor Kazal type 1 (SPINK1) in Prostate Cancer. Med chem 4: 725-728. doi:10.4172/2161-0444.1000220

27. Goldstein AS, Zong Y, Witte ON (2011) A two-step toward personalized therapies for prostate cancer. Sci Transl Med 3: 72ps7.

28. Scheving LA (1983) Primary amino acid sequence similarity between human epidermal growth factor-urogastrone, human pancreatic secretory trypsin inhibitor, and members of porcine secretin family. Arch Biochem Biophys 226 : 411-413.

29. Ozaki N, Ohmuraya M, Hirota M, Ida S, Wang J,et al. (2009) Serine protease inhibitor Kazal type 1 promotes proliferation of pancreatic cancer cells through the epidermal growth factor receptor. Mol Cancer Res 7: 1572-1581.

30. Thiery JP (2002) Epithelial-mesenchymal transitions in tumour progression. Nat Rev Cancer 2: 442-454.

31. Grupp K, Diebel F, Sirma H, Simon R, Breitmeyer K, et al. (2013) SPINK1 expression is tightly linked to 6q15- and 5q21-deleted ERG-fusion negative prostate cancers but unrelated to PSA recurrence. Prostate 73: 1690-1698.

32. Anderson DR, Grillo-Lopez A, Varns C, Chambers KS, Hanna N (1997) Targeted anti-cancer therapy using rituximab, a chimaeric anti-CD20 antibody
(IDEC-C2B8) in the treatment of non-Hodgkin's B-cell lymphoma. Biochem Soc Trans 25: 705-708.

33. Druker BJ, Tamura S, Buchdunger E, Ohno S, Segal GM, et al. (1996) Effects of a selective inhibitor of the Abl tyrosine kinase on the growth of Bcr-Abl positive cells. Nat Med 2: 561-566.

34. Palanisamy N, Ateeq B, Kalyana-Sundaram S, Pflueger D, Ramnarayanan K, et al. (2010) Rearrangements of the RAF kinase pathway in prostate cancer, gastric cancer and melanoma. Nat Med 16: 793-798.

35. Schlomm T, Kirstein P, Iwers L, Daniel B, Steuber T, et al. (2007) Clinica significance of epidermal growth factor receptor protein overexpression and gene copy number gains in prostate cancer. Clin Cancer Res 13: 6579-6584.

36. Slovin SF, Kelly WK, Wilton A, Kattan M, Myskowski P, et al. (2009) Antiepidermal growth factor receptor monoclonal antibody cetuximab plus Doxorubicin in the treatment of metastatic castration-resistant prostate cancer. Clin Genitourin Cancer 7: E77-82.

37. Scott AM, Wolchok JD, Old LJ (2012) Antibody therapy of cancer. Nat Rev Cancer 12: 278-287. 